\title{
Nanostructured Electrodes For Biosensing: An Innovative Technology With Huge Potential Applications
}

\author{
Giuseppe Aiello* and Rosalinda Inguanta \\ Dipartimento di Ingegneria, Università di Palermo, Italy \\ *Corresponding author: Giuseppe Aiello, Dipartimento di Ingegneria, Università di Palermo, Viale delle Scienze, Italy. \\ To Cite This Article: Giuseppe Aiello. Nanostructured Electrodes For Biosensing: An Innovative Technology With Huge Potential Applications. Am \\ J Biomed Sci \& Res. 2019 - 5(3). AJBSR.MS.ID.000911. DOI: 10.34297/AJBSR.2019.05.000911.
}

Received: 眥 September 19, 2019; Published: 觜 September 23, 2019

\section{Mini Review}

Nanomaterials is a recent technology with a wide landscape of potential applications in several fields. In particular, recent researches have demonstrated their appropriateness for biosensing applications in the clinical diagnosis and prevention of several important pathologies. Recent developments in the application of Carbon-based nanomaterials such as carbon nanotubes (CNT) and graphene electrodes have proven their outstanding capabilities of detecting the presence of electroactive compounds in biological fluids, thanks to their excellent electrical conductivity, large surface area and low cost. The structure of graphene is two-dimensional with the carbon atoms forming hexagonal lattices, providing a large surface area for chemical detection. The ability of rapid electron transfer kinetics at the basal planes of graphene sheets act as nanoconnectors and thus electrically facilitates the heterogeneous electron transfer between the biomolecules and electrode substrate, resulting in excellent sensing performance. As one of the most important applications of biosensors, Dopamine (DA) detection in biological fluids is gaining a lot of attention both from researchers and practitioners. In fact, in the last years, dopamine concentration in human body fluids has been related to some neurodegenerative diseases, such as Parkinson and Alzheimer's diseases [1]. The possibility to have a bio-marker for these disease is of extreme importance because, disease related with dementia, are diagnosed when they are already developed and their management become almost impossible. The possibility to continuously monitor DA level in fluids, such as blood and urine, could accelerate the early diagnosis of these diseases.

The principal analytical method to detected dopamine is High Pressure Liquid Chromatrography (HPLC), but this technique does not allow any kind of real time or in situ analysis and, furthermore, is highly expensive and hard to use [2,3]. To achieve a real-time screening of DA, electrochemical sensors are perfect candidates [4]. There is currently a huge potential market for sensing services and devices based on such technology in the area $f$ healthcare services for third-age and personalized assistance. In Europe (EU28), currently $17 \%$ of the population, or 85 million people, are 65 years or older. Projections show that in $2030,23 \%$ or 125 million people, and in $2060,30 \%$ or 155 million people, will be 65 years or older. The ongoing demographic ageing is a real challenge to individuals and families, institutions, healthcare and social care systems. Demographic ageing, therefore, opens up for new initiatives and opportunities for active and healthy ageing, and in such context, Information and Communication Technologies (ICT) can play a decisive role. Modern care, in fact, is based on the paradigm of patient-centric personalized medicine, and on a healthcare path starting with investigations, diagnosis and, if judged necessary, treatment. The production of low-cost devices based on nanostructured biosensors is thus a huge market opportunity, considering the current expansion of the target market, which, for example accounted for $\$ 4.08$ billion in 2017 for Global Parkinson's Disease Treatment Market, and is expected to reach $\$ 7.93$ billion by 2026 growing at a CAGR of $7.7 \%$. The exploitation of the outstanding properties of nanomaterials in biosensing devices, this technology can represent a disruptive innovation in the early of several important pathologies, thus increasing the probability of healing and improving the quality of life of millions of patients all over the world [5].

\section{References}

1. OL Lopez, SRWisnieski, JT Becker, F Boller,ST De Kosky (1997) Extrapyramidal signs in patients with probable Alzheimer disease. Arch Neur 54(8): 969-975.

2. HX Zhao, H Mu, YH Bai, H Yu, YM Hu (2011) A rapid method for the determination of dopamine in porcine muscle by pre-column derivatization and HPLC with fluorescence detection. J Pharmac Anal 1(3): 208-212.

3. D Chatterjee, R Gerlai (2009) High precision liquid chromatography analysis of dopaminergic and serotoninergic responses to acute alcohol exposure in zebrafish. Behav Brain Res 200(1): 208-213. 
4. B Patella, C Sunseri, R Inguanta (2018) Nanostructured Based Electrochemical Sensors. J Nanoscie Nanotech 18: 1-12.

5. B Patella, A Sortino, G Aiello, C Sunseri, R Inguanta (2019) Reduced graphene oxide decorated with metals nanoparticles electrode as electrochemical sensor for dopamine. 2019 IEEE International Conference on Flexible and Printable Sensors and Systems (FLEPS), Glasgow, United Kingdom p. 1-3. 